\title{
Balanço de nitrogênio em cordeiros alimentados com rações contendo diferentes teores de concentrado
}

\section{Nitrogen balance in lambs fed diet containing different levels of concentrate}

\author{
Luiz Juliano Valério Geron ${ }^{1 *}$; Fabiana Gomes da Costa ${ }^{2}$; Ruth Helerene Espinosa \\ Santos $^{3}$; Jocilaine Garcia ${ }^{1}$; Raquel Joana Trarutmann-Machado ${ }^{4}$; \\ Maria Isabel Leite da Silva²; Lúcia Maria Zeoula ${ }^{5}$; Dilma Alves Silva ${ }^{1}$
}

\begin{abstract}
Resumo
Avaliou-se o consumo, a produção fecal e urinária de nitrogênio e o balanço de nitrogênio $(\mathrm{BN})$ em cordeiros alimentados com diferentes teores de concentrado $(20,0 \%, 40,0 \%, 60,0 \%$ e $80,0 \%)$ na região sudoeste do estado de Mato Grosso. Foram utilizados quatro cordeiros sem raça definida (SRD), inteiros, com peso corporal (PC) médio de 19,3 $\mathrm{kg} \pm 2,1$ alocados em gaiolas de metabolismo, alimentados duas vezes ao dia. O concentrado foi composto por grão de milho moído e farelo de soja e o volumoso fornecido foi silagem de milho. Foi utilizado um delineamento experimental em quadrado latino 4X4. As sobras, as fezes e a urina foram coletadas diariamente durante seis dias em cada período de coleta. Cada período experimental teve duração de 20 dias. Os dados de consumo de nitrogênio $(\mathrm{N}), \mathrm{N}$ fecal, $\mathrm{N}$ urinário, $\mathrm{N}$ absorvido e $\mathrm{BN}$ expressos em $\mathrm{g} \mathrm{dia}^{-1}$; \% do nitrogênio consumido (NC) e gramas por quilograma de peso metabólico $\left(\mathrm{g} \mathrm{kg}^{0,75-1}\right)$ foram submetidos á análise de variância (ANOVA) e testados utilizando equação de regressão a $5 \%$ de probabilidade. Foi observado que a inclusão de diferentes teores de concentrado fornecidos aos cordeiros sobre o consumo de $\mathrm{N}, \mathrm{N}$ urinário e $\mathrm{N}$ absorvido em $\mathrm{g}$ dia $^{-1}, \mathrm{~g} \mathrm{~kg}^{0,75-1}$ e em $\%$ do NC apresentou um efeito quadrático $(\mathrm{P}<0,05)$ com ponto de máximo obtido para a inclusão de $40,0 \%$ de concentrado nas rações experimentais. Para o nitrogênio fecal em $\mathrm{g} \mathrm{dia}^{-1}, \mathrm{~g}$ $\mathrm{kg}^{0,75-1}$ e em \% NC e para o balanço de nitrogênio, a inclusão de concentrado na dieta alterou $(\mathrm{P}<0,05)$ de maneira linear decrescente com melhores resultados obtidos para os teores de inclusão de $20,0 \%$ a $40,0 \%$ de concentrado na dieta. Assim, conclui-se que o teor de 40,0\% de concentrado na alimentação de cordeiros propicia os melhores valores de consumo de nitrogênio, nitrogênio urinário e nitrogênio absorvido em $\mathrm{g} \mathrm{dia}^{-1}$, $\mathrm{g} \mathrm{kg}^{0,75-1}$ e em \% do nitrogênio consumido, além de proporcionar resultados satisfatórios para os valores de nitrogênio fecal e balanço de nitrogênio expressos em $\mathrm{g} \mathrm{dia}^{-1}, \mathrm{~g} \mathrm{~kg}^{0,75-1}$ e em $\%$ do nitrogênio consumido.
\end{abstract}

Palavras-chave: Carboidratos, consumo, farelo de soja, urina

\begin{abstract}
Evaluated the nitrogen intake, fecal and urinary nitrogen production and nitrogen balance (NB) in lambs fed levels increasing of concentrate $(20.0 \%, 40.0 \%, 60.0 \%$ and $80.0 \%)$ in the southwestern region of
\end{abstract}

1 Profs., Universidade do Estado de Mato Grosso, UNEMAT, Pontes e Lacerda, MG. E-mail: ljgeron@yahoo.com.br; jo@unemat. br; dilmavet@hotmail.com

2 Discentes, UNEMAT, Pontes e Lacerda, MG. E-mail: fabiana.cta@hotmail.com; mariaisabelmt@hotmail.com

3 Bacharel em Zootecnia, UNEMAT Pontes e Lacerda, MG. E-mail: ruth_zootecnia@hotmail.com

${ }^{4}$ M.e do Instituto de Desenvolvimento Agropecuário do Mato Grosso, INDEA, Pontes e Lacerda, MG. E-mail: raquel_trautmann@ hotmail.com

5 Prof ${ }^{a}$, Universidade Estadual de Maringá, UEM, Maringá, PR. E-mail: 1mzeoula@uem.br

* Autor para correspondência 
Mato Grosso. Were used four lambs mongrel (SRD), intact, with body weight (BW) averaged $19.3 \pm$ $2.1 \mathrm{~kg}$ allocated to metabolism cages, fed twice daily, the concentrate was composed of ground corn and soybean and roughage utilized was corn silage. Were used a design in latin square 4X4, the leavings, faeces and urine were collected daily for six days, in each collection period. Each experimental period lasted 20 days. Intake data of nitrogen $(\mathrm{N})$, fecal $\mathrm{N}$, urinary $\mathrm{N}$, absorbed $\mathrm{N}$ and $\mathrm{NB}$ expressed in $\mathrm{g}$ day $^{-1}, \%$ of nitrogen intake (NI) and grams per kilogram of metabolic weight $\left(\mathrm{g} \mathrm{kg}^{0,75^{-1}}\right)$ were undergo analysis of variance (ANOVA) and tested using regression equation at 5\% probability. It was observed that the inclusion of different levels of concentrate supplied the lambs, on the intake $\mathrm{N}, \mathrm{N}$ absorbed and urinary in $\mathrm{g} \mathrm{day}^{-1}, \mathrm{~g} \mathrm{~kg}^{0.75-1}$ and \% NI presented a quadratic effect $(\mathrm{P}<0.05)$ with the maximum point obtained for the inclusion of $40.0 \%$ concentrate on experimental diets. For fecal nitrogen in $\mathrm{g} \mathrm{day}^{-1}, \mathrm{~g}$ $\mathrm{kg}^{0.75-1}$ and $\% \mathrm{NI}$ and nitrogen balance (NB), the inclusion of concentrate in the diet changed $(\mathrm{P}<0.05)$ linearly decreasing with better results for inclusion levels of $20.0 \%$ to $40.0 \%$ concentrate diet. Thus, it is concluded that the level of $40.0 \%$ concentrate in the diet of lambs provides the best values of nitrogen intake, urinary nitrogen and nitrogen absorbed in $\mathrm{g} \mathrm{day}^{-1}, \mathrm{~g} \mathrm{~kg}^{0.75-1}$ and in $\%$ of nitrogen intake, and provide satisfactory results for the values of fecal nitrogen and nitrogen balance expressed in $\mathrm{g}$ day ${ }^{1}, \mathrm{~g}$ $\mathrm{kg}^{0.75-1}$ and in $\%$ of nitrogen consumed.

Key words: Carbohydrates, intake, soybean meal, urine

\section{Introdução}

A criação de ovinos no Brasil se torna cada vez mais ampla, apesar da atividade ocupar pequeno espaço na mídia. Atualmente existem poucos ovinocultores que se dedicam à criação de animais de alto padrão genético para a reprodução e normalmente os ovinos são criados juntamente com bovinos, ocupando o mesmo espaço e as mesmas pastagens, geralmente de grande extensão. $\mathrm{O}$ que não é diferente na região Sudoeste do Mato Grosso em que a ovinocultura ainda é pouca expressiva, porém existem no Estado alguns empreendimentos com investimentos que fazem da criação de ovinos uma atividade econômica, praticada com níveis satisfatórios de tecnologia e que está cada vez mais se expandindo (GERON et al., 2012a).

Revisão realizada por Souza (2009) enfatizou que a ovinocultura não está com o mesmo nível de maturidade estratégica e de negócios de outros setores da pecuária como a avicultura, a suinocultura e mesmo a bovinocultura. Praticamente não há integração contratual entre produtores e agroindústrias, os sistemas de produção e o padrão tecnológico não estão formalizados em processos e a pesquisa existente não chega satisfatoriamente ao setor produtivo.

De acordo com Menezes (2011), os sistemas de produção que visam custo mínimo podem utilizar quantidades de alimentos que atinjam as exigências nutricionais dos animais de produção a fim de se evitar o desperdício. O conhecimento sobre o consumo e a digestibilidade dos nutrientes presentes nas dietas podem auxiliar na determinação de quais alimentos podem ser utilizados em conjunto, buscando alcançar a interação positiva e atingir as exigências nutricionais de maneira adequada além de propiciar a redução do custo da alimentação (GERON et al., 2012a; GERON et al., 2012b).

Os animais ruminantes, de modo geral, apresentam características de fermentação extremamente eficientes para os carboidratos de reserva e fibrosos, além das proteínas. Desta maneira, a intensidade de fermentação dos carboidratos e a degradação da proteína podem influenciar a correta manutenção do processo de fermentação existente no rúmen (ZEOULA et al., 2006).

De acordo com Fu et al. (2001), a necessidade de proteína de um animal pode ser definida como o menor nível de ingestão protéica da dieta, a qual irá equilibrar as perdas de nitrogênio para o organismo em equilíbrio de energia e em atividade física moderada. Desta forma, a qualidade, quantidade e a digestibilidade das proteínas são importantes, uma vez que essas são indicadores do fornecimento de quantidades significativas de aminoácidos essenciais 
e estão envolvidas na retenção de nitrogênio no organismo animal.

O balanço de nitrogênio (BN) corresponde á diferença entre a quantidade de nitrogênio ingerido e o valor excretado pela urina e fezes (ZEOULA et al., 2003). Este valor pode ser positivo, negativo ou igual a zero (representando o equilíbrio). De acordo com Berchielli, Pires e Oliveira (2011), os procedimentos para determinação do nitrogênio ingerido e o nitrogênio perdido (fezes e urina) pode gerar erros de estimativa do BN. Nas situações onde ocorre carência protéica pode haver redução intracelular do teor de aminoácidos livres, tanto essencial como não essencial e diminuição da excreção urinaria do nitrogênio, resultando em menor perda orgânica de proteína.

O balanço dos compostos nitrogenados pode refletir na resposta produtiva por meio dos produtos finais absorvidos e da extensão das perdas excretadas, além de estar correlacionado positivamente, com a concentração de uréia encontrada na urina, com as concentrações de nitrogênio no plasma e com a ingestão de nitrogênio dos animais (VAN SOEST, 1994).

Segundo Zeoula et al. (2006), o maior valor observado para o $\mathrm{BN}$ para as rações contendo maior teor de concentrado pode ser decorrentes da maior ingestão e maior digestibilidade das dietas que contem maior teor de ingredientes e nutrientes solúveis, os quais normalmente estão presentes nos alimentos concentrados.

Segundo Zeoula et al. (2003), o aumento dos teores de PB da ração, do consumo de nitrogênio e do tipo de fonte de nitrogênio utilizado pode refletir na relação entre o $\mathrm{N}$ excretado pelas vias urinária e fecal. Além disso, o excesso de amônia resultante da rápida hidrólise ruminal da uréia ou de fontes de rápida degradação ruminal e sua posterior absorção pela parede ruminal pode aumentar a excreção de $\mathrm{N}$ pela via urinária, na forma de uréia.

De acordo com Moreno et al. (2010), o aumento do teor de concentrado, independente do tipo de volumoso, refletiu em maior absorção e retenção de nitrogênio, pois, enquanto o consumo e a digestibilidade relacionam-se mais com proporção de volumoso na dieta, o BN é altamente influenciado pelo teor de concentrado na dieta.

Desta maneira, objetivou-se avaliar os diferentes teores de concentrado na alimentação de ovinos sobre o consumo, produção fecal e urinária, absorção e retenção de nitrogênio $(\mathrm{N})$ na região Sudoeste do estado de Mato Grosso.

\section{Material e Métodos}

O estudo foi conduzido na Universidade do Estado de Mato Grosso (UNEMAT), no Campus Universitário de Pontes e Lacerda no Setor de Metabolismo Animal (SeMA) e Laboratório de Análise de Alimentos e Nutrição Animal (LAANA) pertencentes à UNEMAT, situados a 15'19'05" de latitude Sul e 59 13'26" de longitude Oeste e a uma altitude de 295 metros.

Foram utilizados quatro cordeiros inteiros, sem raça definida (SRD) com peso corporal (PC) inicial médio de 19,3 $\pm 2,1 \mathrm{~kg}$. Os mesmos foram alojados em gaiolas de metabolismo contendo comedouros e bebedouros individuais. As rações experimentais foram fornecidas duas vezes ao dia. Os cordeiros foram everminados via oral com produto a base de "closantel", quinze dias antes do início do período experimental.

Foi utilizado delineamento experimental em quadrado latino (4X4) com quatro animais, quatro períodos e quatro rações experimentais com diferentes teores de inclusão de concentrado $(20,0 \% ; 40,0 \% ; 60,0 \%$ e $80,0 \%)$ na alimentação de cordeiros para avaliar os seus efeitos sobre o balanço de nitrogênio - BN (consumo, produção urinaria e fecal, absorção e retenção de nitrogênio).

$\mathrm{O}$ experimento foi dividido em quatro períodos experimentais com duração de 20 dias cada, na qual, 14 dias foram para adaptação dos animais e 6 dias para coletas de amostras de sobras, urina e 
fezes, tendo o experimento, duração de 80 dias no total. Os períodos de adaptação e de coleta foram realizados com manejo descrito por Silva e Leão (1979).

Para a confecção das rações experimentais, foram utilizados como alimentos concentrados o grão de milho moído (MG) e o farelo de soja (FS) e como alimento volumoso a silagem de milho (SM) - (Tabela 1). As rações foram balanceadas para apresentar 13,0\% de proteína bruta (isoprotéica - NRC, 1985) e conforme ocorreu a inclusão do concentrado nas rações experimentais, o valor de nutrientes digestíveis totais foi crescente (Tabela 2).

Tabela 1. Composição bromatológica dos alimentos experimentais.

\begin{tabular}{lcccccccc}
\hline \multirow{2}{*}{ Alimento } & \multirow{2}{*}{ MS \% } & \multicolumn{7}{c}{ Notrientes expressos em \% da MS } \\
\cline { 6 - 9 } & & MO & PB & EE & FDN & FDA & MM & NDT $^{1}$ \\
\hline SM & 24,26 & 93,24 & 7,30 & 2,25 & 57,43 & 38,48 & 6,76 & 63,13 \\
MG & 89,98 & 98,45 & 9,10 & 4,20 & 15,57 & 5,88 & 1,55 & 86,44 \\
FS & 88,62 & 92,71 & 50,00 & 1,16 & 14,44 & 10,41 & 7,29 & 88,73 \\
\hline
\end{tabular}

SM: silagem de milho; MG: milho grão moído; FS: farelo de soja; MS: matéria seca; MO: matéria orgânica; PB: proteína bruta; EE: extrato etéreo; FDN: fibra em detergente neutro; FDA: fibra em detergente ácido; MM: matéria mineral e ${ }^{1}$ NDT: nutrientes digestíveis totais, valores obtidos de acordo com Valadares Filho et al. (2006).

Fonte: Elaboração dos autores.

Foi fornecido diariamente $10 \mathrm{~g}$ de mistura mineral por animal, adicionado diretamente sobre o concentrado no momento do fornecimento das dietas, ou seja, duas vezes ao dia ( $5 \mathrm{~g}$ de sal refeição ${ }^{-1}$ animal $\left.^{-1}\right)$. A composição química do sal mineral segundo o fabricante foi de $120 \mathrm{~g}^{\mathrm{de}} \mathrm{Ca} \mathrm{kg}^{-}$ 1; $85 \mathrm{~g} \mathrm{de} \mathrm{P} \mathrm{kg}^{-1} ; 16 \mathrm{~g}_{\mathrm{de}} \mathrm{S} \mathrm{kg}^{-1} ; 148 \mathrm{~g} \mathrm{de} \mathrm{Na} \mathrm{kg}^{-1} ; 50$ $\mathrm{mg}$ de $\mathrm{Co} \mathrm{kg}^{-1} ; 500 \mathrm{mg}$ de $\mathrm{Cu} \mathrm{kg}^{-1} ; 16$ mg de $\mathrm{Se} \mathrm{kg}^{-1}$ e $4.800 \mathrm{mg}^{\mathrm{de}} \mathrm{Zn} \mathrm{kg}^{-1}$.

O fornecimento das rações experimentais foi realizado ad libtum de maneira que, diariamente, houvesse aproximadamente $10 \%$ de sobras, sendo a mesma dividida em duas refeições ao dia, sendo fornecidas às $7 \mathrm{~h}$ e às $17 \mathrm{~h}$. Diariamente, antes do primeiro trato, foi realizado o monitoramento das sobras. Os animais tiveram acesso à água por meio de bebedouros individuais.

Inicialmente foram coletadas amostras da silagem de milho em diferentes pontos do silo, para determinar o teor em matéria seca. No transcorrer do experimento foram coletadas amostras das sobras de ração por animal período ${ }^{-1}$ tratamento $^{-1}$.
Para proceder a coleta total de fezes, em cada cordeiro foi adaptada uma sacola de napa durante o período experimental. As fezes de cada animal foram pesadas diariamente pela manhã e homogeneizadas, sendo retiradas amostras compostas, correspondentes a $10 \%$ de seu peso total. As amostras foram acondicionadas em sacos plásticos, identificadas por animal e período experimental e armazenadas em freezer com temperatura a $-10^{\circ} \mathrm{C}$, Para a coleta total de urina, foram utilizados baldes plásticos cobertos com telas, para evitar contaminação com pêlos, ração e fezes, sendo os baldes alocados em baixo das gaiolas de metabolismo. Foi adicionado em cada balde, $20 \mathrm{~mL}$ de acido clorídrico $(\mathrm{HCl})$ (1:1) para evitar a volatilização do N (nitrogênio) como também a possível fermentação. A coleta de urina foi realizada diariamente sempre no mesmo horário, ou seja, pela manhã. Amostras de $10 \%$ do total de urina foram acondicionadas em um único frasco de vidro, devidamente identificado por animal, em cada período experimental. 
Tabela 2. Composição percentual e bromatológica das rações experimentais.

\begin{tabular}{lcccc}
\hline Alimentos & \multicolumn{4}{c}{ Teores de concentrado nas rações experimentais } \\
\cline { 2 - 5 } & $20,0 \%$ & $40,0 \%$ & $60,0 \%$ & $80,0 \%$ \\
\hline Composição percentual das rações experimentais & & & & \\
Silagem de milho & 80 & 60 & 40 & 20 \\
Grão de milho moído & 8 & 28,5 & 49 & 69,5 \\
Farelo de soja & 12 & 11,5 & 11 & 10,5 \\
Total & 100,0 & 100,0 & 100,0 & 100,0 \\
Composição bromatológica (\% na MS) das rações experimentais & & & \\
Matéria seca (MS) & 37,24 & 50,39 & 63,54 & 76,69 \\
Proteína bruta (PB) & 12,57 & 12,72 & 12,88 & 13,03 \\
Fibra em detergente neutro (FDN) & 48,92 & 40,56 & 32,19 & 23,82 \\
Nutrientes digestíveis totais (NDT) & 68,07 & 72,72 & 77,37 & 82,02 \\
\hline
\end{tabular}

Fonte: Elaboração dos autores.

Após o período experimental de coleta, as amostras de alimentos, sobras e fezes foram secas em estufa a $55{ }^{\circ} \mathrm{C}$ por 72 horas, concomitantemente foram processadas em moinho de facas utilizandose peneira de crivos de $1 \mathrm{~mm}$, sendo em seguida homogeneizadas em quantidades iguais, com base no peso seco, para formar amostras compostas de fezes e sobras por animal período ${ }^{-1}$ ração experimental ${ }^{-1}$.

Foi realizada a determinação do teor de $\mathrm{N}$ dos alimentos estudados, das sobras, urina e fezes, em que os mesmos foram obtidos pelo método semi-microKjeldahl, usando 6,25 como fator de conversão para PB. A análise para determinar a matéria mineral (MM) foi realizada através do método por incineração em mufla a $600^{\circ} \mathrm{C}$, obtendo-se o valor de matéria orgânica (MO) por diferença e o teor de extrato etéreo (EE) foi determinado pela extração por lavagem com éter de petróleo, segundo citações de Silva e Queiroz (2002). Para a determinação da concentração da fibra em detergente neutro (FDN) e ácido (FDA) foram realizadas análises pelo método de Van Soest, Robertson e Lewis (1991).

$\mathrm{O}$ teor de nitrogênio $(\mathrm{N})$ na urina foi calculado pelo método Kjeldahl, descrito por Silva e Queiroz (2002), e o balanço de N, ou nitrogênio retido foi obtido utilizando-se a fórmula: $\mathrm{BN}=[(\mathrm{N}$ fornecido $\mathrm{g}-\mathrm{N}$ das sobras $\mathrm{g})-(\mathrm{N}$ nas fezes $\mathrm{g}+\mathrm{N}$ na urina g)] conforme descrito por Zeoula et al. (2006). O nitrogênio absorvido foi calculado pela equação: $\mathrm{N}$ absorvido $=[(\mathrm{N}$ fornecido $\mathrm{g}-\mathrm{N}$ da sobras $\mathrm{g})-(\mathrm{N}$ nas fezes $\mathrm{g})$ ] e o $\mathrm{N}$ consumido pela equação: $\mathrm{N}$ cons $=[(\mathrm{N}$ fornecido $\mathrm{g}-\mathrm{N}$ nas sobras $\mathrm{g})]$, de acordo com Moreno et al. (2010).

A análise das variáveis estudadas foi interpretada por meio de análise de variância no programa Sistema de Análise Estatística e Genética - SAEG (UNIVERSIDADE FEDERAL DE VIÇOSA, 1997) e as diferenças observadas para as variáveis estudadas (consumo, produção urinaria e fecal, absorção e retenção de nitrogênio) em relação aos teores de inclusão de concentrado nas rações foram submetidos à equação de regressão a 5\% de significância.

\section{Resultados e Discussão}

Para o consumo de nitrogênio $(\mathrm{N})$ expresso em $\mathrm{g}$ $\mathrm{dia}^{-1}$ e em $\mathrm{g} \mathrm{kg}^{0,75-1}$ dos cordeiros foi observado efeito quadrático $(\mathrm{P}<0,05)$ conforme houve a inclusão dos diferentes teores de concentrado (Figura 1).

Segundo Geron et al. (2013), o consumo de matéria seca e proteína bruta foi influenciado de maneira quadrática $(\mathrm{P}<0,05)$ com a inclusão de $20,0 \% ; 40,0 \% ; 60,0 \%$ e $80,0 \%$ de concentrado na alimentação de cordeiros com ponto de máximo 
observado para os níveis de 35,0 e $37,0 \%$ de concentrado, respectivamente. Este fato pode colaborar na compreensão do comportamento quadrático $(\mathrm{P}<0,05)$ observado no presente estudo para o consumo de $\mathrm{N}$ expresso em $\mathrm{g} \mathrm{dia}^{-1}$ e $\mathrm{g} \mathrm{kg}^{0,75}$
-1, os quais apresentaram ponto de máximo para os níveis de $38,0 \%$ e 40,0\%, obtido pela equações de regressões, com valores de $12,62 \mathrm{~g} \mathrm{dia}^{-1}$ e 1,53 $\mathrm{g}$ $\mathrm{kg}^{075-1}$, respectivamente.

Figura 1. Consumo de nitrogênio - $\mathrm{N}$ em $g$ dia $^{-1}(\mathrm{~A})$ e $\mathrm{g} \mathrm{kg}^{0,75-1}$ (B) dos cordeiros alimentados com diferentes teores de concentrado nas rações experimentais.

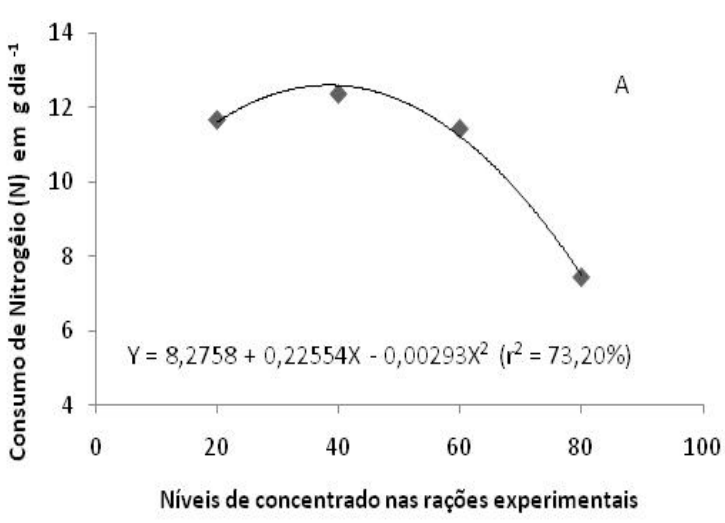

Fonte: Elaboração dos autores.

Estudo realizado por Ezequiel et al. (2000) demonstrou que o consumo de $\mathrm{N}$ e o $\mathrm{BN} \mathrm{g}_{\text {dia }}{ }^{-1}$ não foram alterados $(\mathrm{P}>0,05)$ pelas diferentes fontes de proteína utilizadas na alimentação de ovinos, os mesmos autores ainda relataram que a uniformidade na ingestão de nitrogênio possibilitou evidenciar possíveis diferenças na qualidade das fontes nitrogenadas utilizadas, demonstrando que as perdas de $\mathrm{N}$ nas fezes foram superiores para as dietas com farelo de algodão em relação aquelas com uréia.

De acordo com Moreno et al. (2010), o teor de concentrado na dieta de ovinos aumentou $(\mathrm{P}<0,05)$ o consumo de $\mathrm{N}$ em $\mathrm{g} \mathrm{dia}{ }^{-1}$. Os autores observaram que para dietas com $40,0 \%$ de concentrado, o consumo de $\mathrm{N}$ foi de $27,26 \mathrm{~g} \mathrm{dia}^{-1}$, porém os animais alimentados com $60,0 \%$ de concentrado apresentaram um consumo de $\mathrm{N}$ de $32,60 \mathrm{~g} \mathrm{dia}^{-1}$, valores superiores ao observado no presente estudo, os quais variaram de 7,43 a 12,38 $\mathrm{g} \mathrm{dia}^{-1}$ (Figura 1). Este maior consumo de $\mathrm{N}$ observado no trabalho de

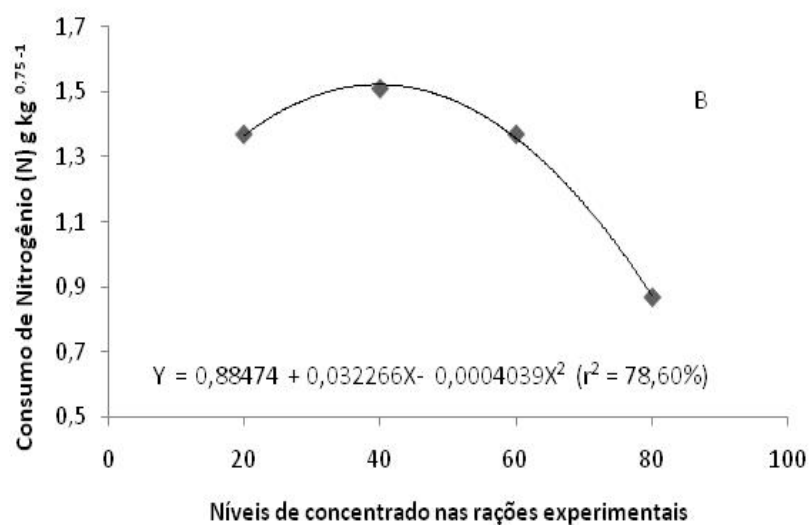

Moreno et al. (2010) em relação ao presente estudo pode ser devido ao maior teor de $\mathrm{PB}$ das rações experimentais, com média de $19,0 \%$, em relação aos $13,0 \%$ PB no presente estudo, além da utilização de fonte de nitrogênio não protéico (uréia) por esses autores.

Da mesma forma Bringel et al. (2011), ao avaliarem a substituição do capim elefante pela torta de dendê nos níveis de $0,0 \% ; 20,0 \% ; 40,0 \% ; 60,0 \%$ e $80,0 \%$ na alimentação de borregos observaram que os animais apresentaram comportamento quadrático $(\mathrm{P}<0,05)$ para o consumo de $\mathrm{PB}$ e $\mathrm{N}$ $\mathrm{g} \mathrm{dia}^{-1}$ com a inclusão da torta de dendê na dieta, uma vez que a inclusão deste alimento propiciou aumento no valor de PB da dieta variando de 8,14 a 11,64\%. O consumo de $\mathrm{N}$ observado por estes autores variou de 5,30 a 15,03 $\mathrm{g} \mathrm{dia}^{-1}$, sendo o valor máximo observado para o teor de $40,0 \%$ de torta de dendê (concentrado) na dieta.

Ao avaliar o $\mathrm{N}$ fecal expresso em $\mathrm{g}$ dia $^{-1}$ e $\mathrm{g}$ $\mathrm{kg}^{0,75-1}$ foi observado um efeito linear decrescente 
$(\mathrm{P}<0,05)$ com a inclusão dos diferentes teores de concentrado na alimentação dos cordeiros (Figura 2). Foi estimado que o teor de 20,0\% de concentrado proporcionou o maior valor de $\mathrm{N}$ fecal com valor de 5,57 $\mathrm{g} \mathrm{dia}^{-1}$ e de $0,66 \mathrm{~g} \mathrm{~kg}^{0,75-1}$ (Figura
2). Entretanto, para o $\mathrm{N}$ fecal em $\%$ do nitrogênio consumido $(\mathrm{NC})$ foi observado um comportamento quadrático $(\mathrm{P}<0,05)$ com a inclusão dos teores de concentrado com ponto de mínimo estimado para o teor de $50,0 \%$ de concentrado, o qual propiciou um valor de N fecal de $41,69 \%$ do NC (Figura 2C).

Figura 2. Nitrogênio fecal expresso em $\mathrm{g} \mathrm{dia}^{-1}$ (A), $\mathrm{g} \mathrm{kg}^{0,75-1}$ (B) e em \% nitrogênio consumido - NC (C) dos cordeiros alimentados com os diferentes teores de concentrado nas rações experimentais.
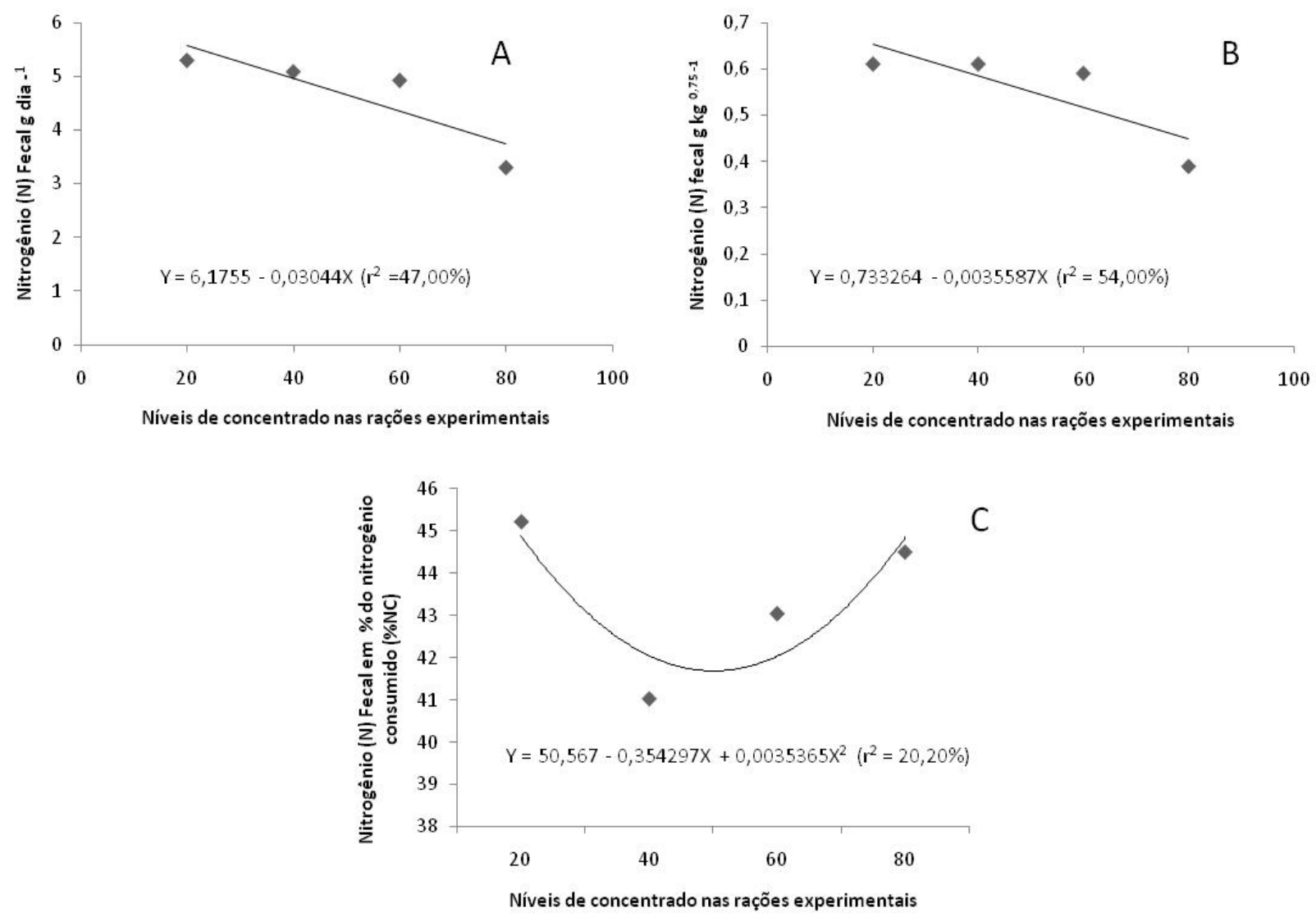

Fonte: Elaboração dos autores.

A quantidade de $\mathrm{N}$ fecal normalmente aumenta com a atividade fermentativa no intestino grosso, devido ao maior aporte de nitrogênio de origem microbiana nas fezes, o que ocorre particularmente quando as dietas são ricas em grãos de cereais, como milho e sorgo (KOZLOSKI, 2002).

Segundo Moreno et al. (2010), o N fecal e o urinário não foram alterados $(\mathrm{P}>0,05)$ pelo teor de concentrado de $40,0 \%$ e $60,0 \%$ na dieta cordeiros, com valores médios de 12,30 e $6,60 \mathrm{~g} \mathrm{dia}^{-1}$, respectivamente. Segundo esses autores a perda de nitrogênio pela via fecal e urinária correspondeu a $40,74 \%$ e $22,20 \%$ do $\mathrm{N}$ consumido, respectivamente, indicando que $62,94 \%$ do $\mathrm{N}$ consumido foi perdido nas fezes e urina. Entretanto, no presente estudo foi observado que as menores perdas de $\mathrm{N}$ fecal em relação a \% do NC ocorreram para o nível de $50,0 \%$. Porém os valores do $\mathrm{N}$ fecal apresentaram 
uma amplitude de variação de 5,29 a 3,31 em g dia ${ }^{-1}$, valores menores que os encontrados por Moreno et al. (2010). Este fato provavelmente foi devido ao maior nível de PB fornecido (19,0\%) no estudo desses autores em relação ao presente estudo.

Damasceno et al. (2000) observaram que ovinos alimentados com níveis crescentes de palha de arroz amonizada com uréia $(1,5 \% ; 3,0 \% ; 4,5 \%$; $6,0 \%$ e $7,5 \%$ do peso corporal vivo) mais $200 \mathrm{~g}$ de grão de milho moído por dia, apresentaram um comportamento linear crescente $(\mathrm{P}<0,05)$ para $\mathrm{o}$ $\mathrm{N}$ fecal em $\mathrm{g}$ dia $^{-1}$ conforme aumentou o nível de fornecimento da palha amonizada na dieta dos ovinos. Os autores observaram uma variação no $\mathrm{N}$ fecal de 7,51 a 12,64 $\mathrm{g} \mathrm{dia}^{-1}$ para os diferentes níveis de inclusão de palha de arroz amonizada na dieta, podendo ter ocorrido devido ao fato do aumento no consumo de $\mathrm{N}$ com o crescente nível de palha de arroz amonizada com uréia na dieta. Os valores de consumo de $\mathrm{Ng} \mathrm{dia}^{-1}$ para o estudo de Damasceno et al. (2000) apresentaram comportamento crescente $(\mathrm{P}<0,05)$ com valores variando de 15,27 a $21,01 \mathrm{~g}$ dia $^{-1}$ com a inclusão da palha de arroz amonizada. Porém no presente estudo foi observado que o consumo de $\mathrm{N}$ g dia ${ }^{-1}$ apresentou comportamento quadrático provavelmente influenciado pelo consumo de MS e PB conforme relatado por Geron et al. (2013).

Para $\mathrm{N}$ urinário expresso em $\mathrm{g} \mathrm{dia}^{-1}, \mathrm{~g} \mathrm{~kg}^{0,75-1} \mathrm{e}$ $\% \mathrm{NC}$ (Figura 3 ) dos cordeiros foi observado efeito $(\mathrm{P}<0,05)$ quadrático com a inclusão dos diferentes teores de concentrado na alimentação. Foi estimado que o ponto de máximo para o $\mathrm{N}$ urinário foi obtido para o teor de concentrado de 53,0\% com valores de 4,79 $\mathrm{g} \mathrm{dia}^{-1}$ (Figura 3A) e 0,58 $\mathrm{g} \mathrm{kg}^{0,75-1}$ (Figura $3 \mathrm{~B})$. Esse efeito da perda de $\mathrm{N}$ urinário $\mathrm{g} \mathrm{dia}^{-1}$ no presente estudo pode ser decorrente do maior $(\mathrm{P}<0,05)$ consumo de MS e PB que ocorreram nos teores próximos de 40,0\% (GERON et al., 2013).

Para o $\mathrm{N}$ urinário expresso em $\% \mathrm{NC}$, o ponto de máximo foi estimado para o nível de $60,0 \%$ de concentrado na dieta, apresentando um valor de $5,10 \%$ NC (Figura 3C).
Entretanto, Moreno et al. (2010) observaram que o $\mathrm{N}$ urinário não foi alterado $(\mathrm{P}>0,05)$ pelo teor de concentrado de $40,0 \%$ e $60,0 \%$ na dieta cordeiros, com valores médios de 6,77 e 6,37 g dia${ }^{1}$, respectivamente. Os autores consideraram que a composição da dieta e o uso de uréia, aliada a grande quantidade de carboidratos prontamente disponíveis no rúmen (amido da silagem de milho e sacarose da cana-de-açúcar) podem ter proporcionado melhor utilização das fontes de proteína o que refletiu em maior retenção de nitrogênio.

De acordo com Santos e Pedroso (2011), a amônia presente no rúmen pode ser absorvida pela parede ruminal na sua forma não ionizada $\left(\mathrm{NH}_{3}\right) \mathrm{e}$ na forma ionizada $\left(\mathrm{NH}_{4}^{+}\right)$a amônia não é absorvida. Portanto a redução do valor de $\mathrm{pH}$ do líquido ruminal favorece a ionização da amônia e reduz sua absorção, enquanto que a elevação do pH do líquido ruminal favorece a presença de amônia na forma não ionizada e aumenta a absorção de anomia. Porem no estudo conduzido por Geron et al. (2013), não foi observado diferença $(\mathrm{P}>0,05)$ para o valor do $\mathrm{pH}$ do líquido ruminal em função dos teores de $20,0 \% ; 40,0 \% ; 60,0 \%$ e $80,0 \%$ de concentrado na alimentação de cordeiros. Entretanto foi observado que o $\mathrm{pH}$ do líquido ruminal apresentou um efeito quadrático $(\mathrm{P}<0,05)$ com ponto de mínimo de 6,64 obtido para 4 horas após a alimentação da manhã para todos os teores de concentrado avaliados, indicando que a absorção de amônia pela parede do rúmen pode ser maior no período de 2 a 6 horas após o consumo de MS e PB pelos cordeiros.

Este fato foi confirmado por Geron et al. (2013), os quais observaram que os teores de concentrado na alimentação de cordeiros não alteram $(\mathrm{P}>0,05)$ a concentração de nitrogênio amoniacal $\left(\mathrm{N}^{-\mathrm{NH}_{3}}\right)$ do líquido ruminal com valor médio de $17,61 \mathrm{mg}$ $\mathrm{N}^{-N_{3}} 100 \mathrm{~mL}^{-1}$ de líquido ruminal. Porém os autores observaram comportamento quadrático $(\mathrm{P}<0,05)$ com ponto de máximo para a concentração do $\mathrm{N}_{-} \mathrm{NH}_{3}$ do líquido ruminal de $19,45 \mathrm{mg} \mathrm{N}-\mathrm{NH}_{3}$ $100 \mathrm{~mL}^{-1}$ de líquido ruminal obtido para $3 \mathrm{~h}$ e $42 \mathrm{~min}$ após alimentação da manhã e esta variação pode 
ter contribuído com as flutuações de absorção da amônia pela parede ruminal e terem influenciado as perdas de $\mathrm{N}$ via urina.

Para o balanço de nitrogênio $(\mathrm{BN})$ expresso em $\mathrm{g}$ dia $^{-1}$ foi observado um efeito $(\mathrm{P}<0,05)$ linear decrescente à medida que aumentou a inclusão de concentrado na dieta (Figura 4). Foi estimado que os maiores valores do $\mathrm{BN}$ de 4,5 $\mathrm{g} \mathrm{dia}^{-1}$ (Figura 4A) e de $0,5 \mathrm{~g} \mathrm{~kg}^{0,75-1}$ (Figura 4B) foram obtidos para o teor de $20,0 \%$ de concentrado. Para o $\mathrm{BN}$ expresso em \% NC (Figura 4C), foi observado efeito quadrático $(\mathrm{P}<0,05)$ com ponto de mínimo estimado para a inclusão de $65,0 \%$ de concentrado com valor de $15,77 \% \mathrm{NC}$.
Este efeito linear decrescente observado para o $\mathrm{BN} \mathrm{g} \mathrm{dia}^{-1}$ e $\mathrm{g} \mathrm{kg}^{0,75-1}$, pode ser devido ao menor consumo de MS e PB dos cordeiros com a utilização dos maiores teores de concentrado na dieta segundo Geron et al. (2013).

Entretanto, Mizubuti et al. (2007) observaram que os ovinos alimentados com $60 \%$ de concentrado (feijão guandu) apresentaram maior valor de BN (13,15 $\left.\mathrm{g} \mathrm{dia}^{-1}\right)$ em relação aos alimentados com $40 \%\left(10,29 \mathrm{~g} \mathrm{dia}^{-1}\right)$ e sugeriram que este fato pode estar relacionado ao maior consumo de PB proporcionada pelo alimento concentrado e pela maior digestibilidade da PB da dieta.

Figura 3. Nitrogênio - $\mathrm{N}$ urinário expresso em $\mathrm{g} \mathrm{dia}^{-1}(\mathrm{~A}), \mathrm{g} \mathrm{kg}^{0,75-1}$ (B) e em \% nitrogênio consumido - NC (C), de cordeiros alimentados com diferentes teores de concentrado nas rações experimentais.
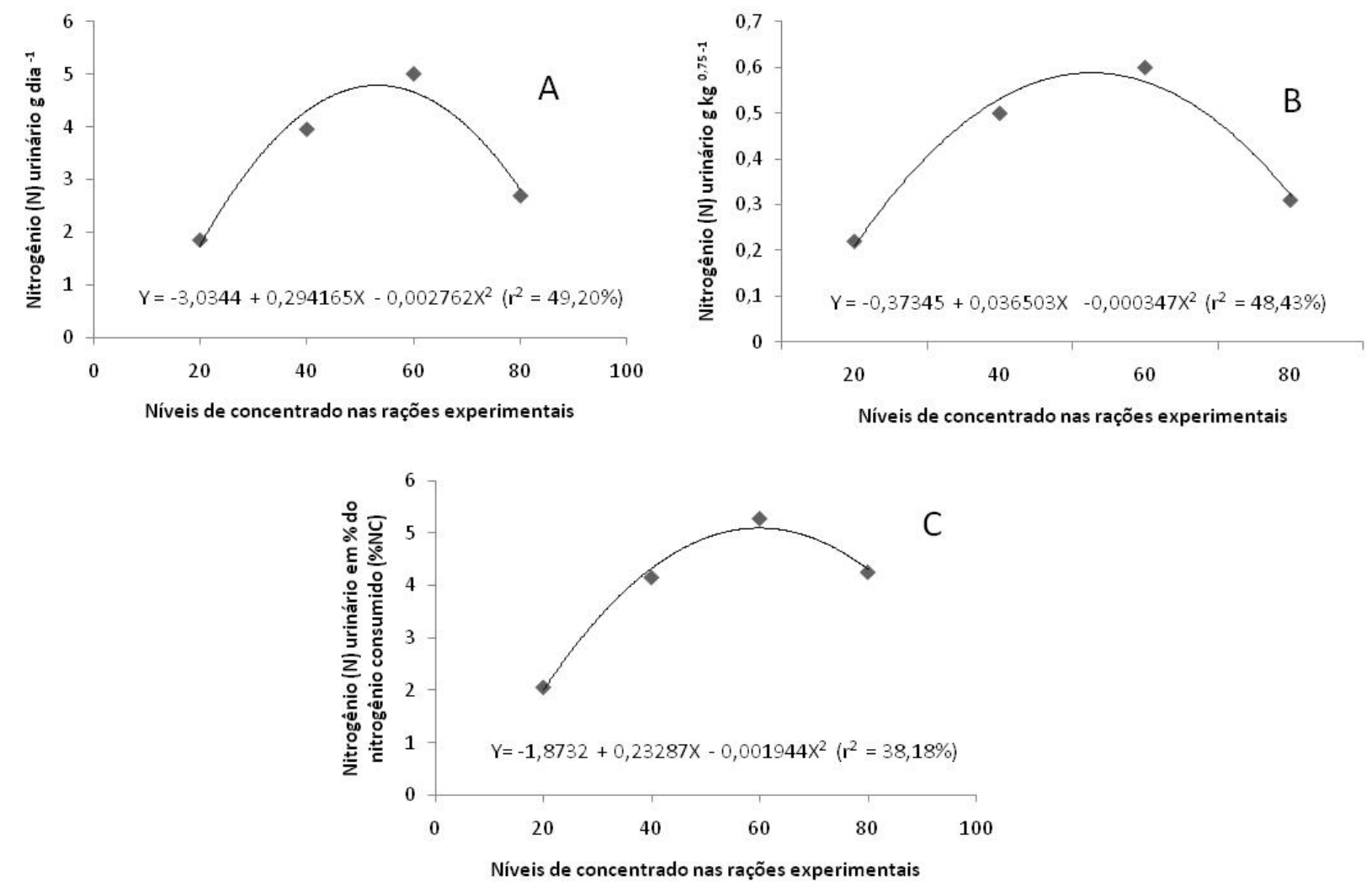

Fonte: Elaboração dos autores. 
Figura 4. Balanço de nitrogênio - $\mathrm{BN}$ expresso em $\mathrm{g} \mathrm{dia}^{-1}(\mathrm{~A}), \mathrm{g} \mathrm{kg}^{0,75-1}$ (B) e em \% nitrogênio consumido - NC (C), de cordeiros alimentados com diferentes teores de concentrado nas rações experimentais.
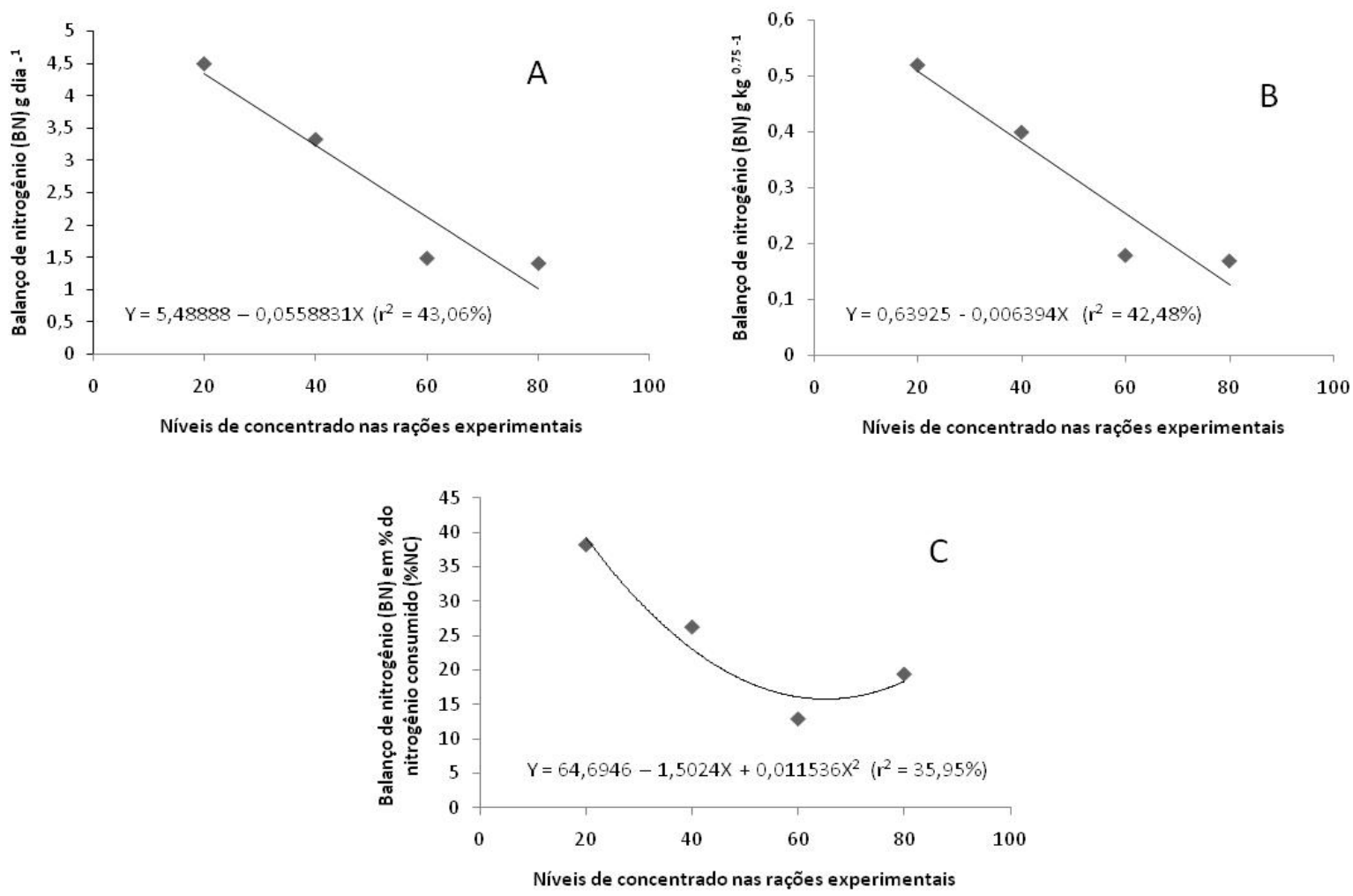

Fonte: Elaboração dos autores.

Estudo conduzido por Geron et al. (2013) demonstrou que o coeficiente de digestibilidade (CD) da $\mathrm{PB}$ para cordeiros apresentou comportamento quadrático $(\mathrm{P}<0,05)$ para a inclusão de diferentes teores de concentrado, com ponto de máximo obtido para o teor de $50,0 \%$ de concentrado, com valor de CD da PB de 58,31\%. Este efeito no CD da PB encontrado por estes autores pode ter ocorrido devido a associação dos efeitos linear decrescente $(\mathrm{P}<0,05)$ do $\mathrm{N}$ fecal em conjunto com as perdas de $\mathrm{N}$ urinário de maneira quadrática $(\mathrm{P}<0,05)$ com a inclusão dos diferentes teores de concentrado na alimentação dos cordeiros.

De acordo com Tibo et al. (2000), o BN aumentou linearmente com os teores de concentrados da dieta variando de 6,4 a $53,3 \mathrm{~g} \mathrm{dia}^{-1}$ e 0,1 a $0,7 \mathrm{~g} \mathrm{~kg}^{0,75-1}$. Os autores verificaram também que o maior aporte de compostos de $\mathrm{N}$ no abomaso resultou em maior retenção de $\mathrm{N}$ no organismo animal.
Porém, Damasceno et al. (2000) não notaram diferença $(\mathrm{P}>0,05)$ para o $\mathrm{BN}$ com a inclusão de diferentes níveis de palha de arroz amonizada. Esses autores sugeriram que a ausência de efeito dos níveis ofertados de palha amonizada sobre o BN poderia ser reflexo da característica do estudo, o qual reflete apenas a diferença entre o $\mathrm{N}$ consumido e o excretado, mas não revela nenhuma informação sobre a distribuição do $\mathrm{N}$ entre tecidos e órgãos, uma vez que o metabolismo animal altera as fontes corporais lábeis de $\mathrm{N}$, dependendo do nível de consumo de $\mathrm{N}$.

Para nitrogênio absorvido em $\mathrm{g} \mathrm{dia}^{-1}$ e $\mathrm{g} \mathrm{kg}^{0,75-1}$ e \% NC (Figura 5) foi observado efeito quadrático $(\mathrm{P}<0,05)$ para os cordeiros alimentados com os diferentes teores de concentrado. Foi estimado que o ponto de máximo para $\mathrm{N}$ absorvido foi de 7,28 $\mathrm{g}$ dia ${ }^{-1}$ obtido para o teor de $41,0 \%$ de concentrado 
(Figura $5 \mathrm{~A}$ ), e de $0,88 \mathrm{~g} \mathrm{~kg}^{0,75-1}$ para o nível $43,0 \%$ de concentrado (Figura 5B). Além disso, o $\mathrm{N}$ absorvido em \% NC apresentou ponto de máximo estimado para o nível de 50,0\% concentrado com um valor N absorvido de 58,3\% NC (Figura 5C).

Figura 5. Nitrogênio - $\mathrm{N}$ absorvido em $\mathrm{g} \mathrm{dia}^{-1}(\mathrm{~A}), \mathrm{g} \mathrm{kg}^{0,75-1}$ (B) e em \% nitrogênio consumido - $\mathrm{NC}$ (C), de cordeiros alimentados com diferentes níveis de concentrado nas rações experimentais.
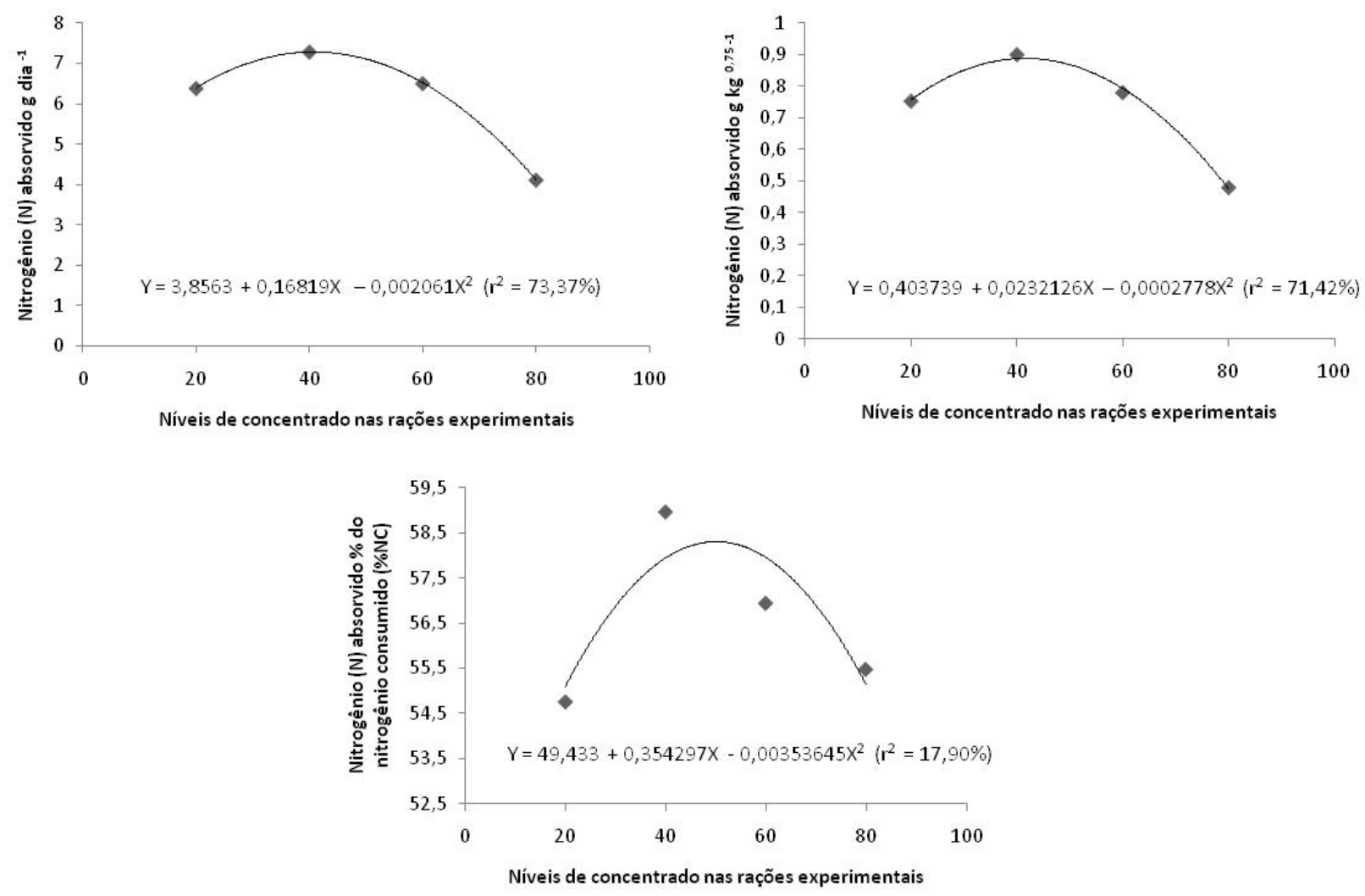

Fonte: Elaboração dos autores.

Este efeito quadrático na absorção de $\mathrm{N}$ em g dia - 1 corrobora com os dados obtidos para o CD da $\mathrm{PB}$, na qual maiores valores foram obtidos para o teor de 50,0\% de concentrado com um CD da PB de $58,31 \%$. Este efeito pode estar correlacionado ao consumo de MS e PB, a perda de $\mathrm{N}$ fecal e ao efeito associativo da relação volumoso:concentrado, o qual pode alterar o processo de fermentação ruminal e consequentemente a síntese de nutrientes pelos microrganismos presentes no rúmen (GERON et al., 2013)

Para a relação do $\mathrm{BN} \mathrm{NC}^{-1}$ e $\mathrm{BN} \mathrm{NA}^{-1}$ (nitrogênio absorvido) foi observado um efeito quadrático
$(\mathrm{P}<0,05)$ com a inclusão do concentrado na alimentação dos cordeiros, com ponto de mínimo estimado para o teor de $65,0 \%$ de concentrado obtendo valores de $0,15 \%$ para o $\mathrm{BN} \mathrm{NC}^{-1}$ e de $0,26 \% \mathrm{BN} \mathrm{NA}^{-1}$ (Figura 6).

Ezequiel et al. (2000) relataram que a retenção de nitrogênio, ou seja, o balanço de nitrogênio (BN) em relação ao nitrogênio absorvido (NA) reflete a utilização do nitrogênio na síntese protéica tissular, seja para formação de novos tecidos, novos sistemas enzimáticos ou para substituir tecidos velhos ou epitélios. A eficiência com que esta atividade é realizada depende fundamentalmente da 
composição do composto nitrogenado que chega aos tecidos, vindos da absorção intestinal. Os resultados obtidos no presente estudo indicam que as proteínas ou outras formas nitrogenadas provenientes dos maiores teores de concentrado de $60,0 \%$ a $80,0 \%$ na alimentação de cordeiros não foram bem utilizadas quando comparadas menores teores $(20,0$ a $40,0 \%)$.

Figura 6. Balaço de nitrogênio (BN) em relação ao nitrogênio consumido $\mathrm{NC}^{-1}$ (A) e $\mathrm{BN}$ em relação ao nitrogênio absorvido $\mathrm{NA}^{-1}$ (B) de cordeiros alimentados com diferentes teores de concentrado.

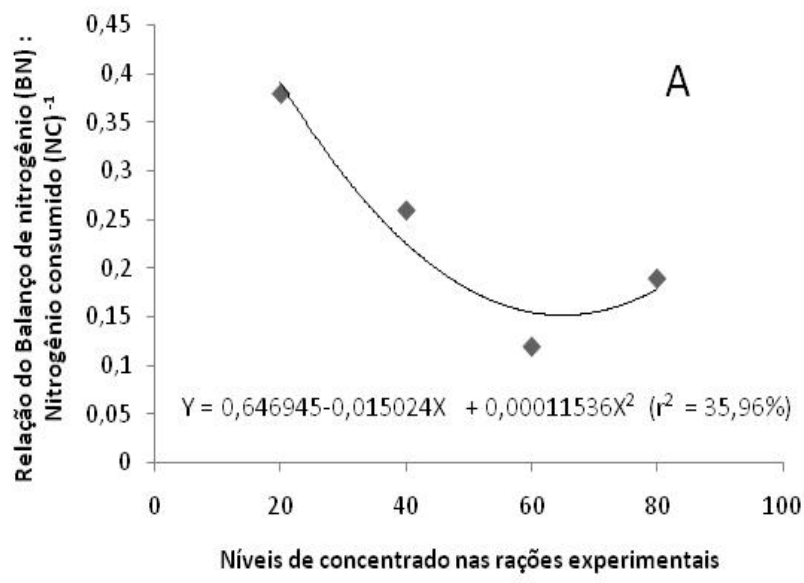

Fonte: Elaboração dos autores.

Segundo Moreno et al. (2010), o estudo sobre o balançodenitrogênioéumindicativodo metabolismo protéico dos animais ruminantes e constitui-se em um importante parâmetro na avaliação de alimentos, o que permite avaliar se o animal encontra-se em equilíbrio quanto aos compostos nitrogenados, o que pode refletir em melhor desempenho e melhor eficiência de utilização da fração protéica da dieta.

Desta maneira, conclui se que a inclusão de $40,0 \%$ de concentrado constituído de milho e farelo de soja na alimentação de cordeiros na região do Sudoeste do Estado de Mato Grosso proporciona os melhores resultados para o consumo de nitrogênio, nitrogênio urinário e nitrogênio absorvido em $\mathrm{g}$ dia1, $\mathrm{g} \mathrm{kg}^{0,75-1}$ e em \% do nitrogênio consumido, além de propiciar resultados satisfatórios para o balanço de nitrogênio.

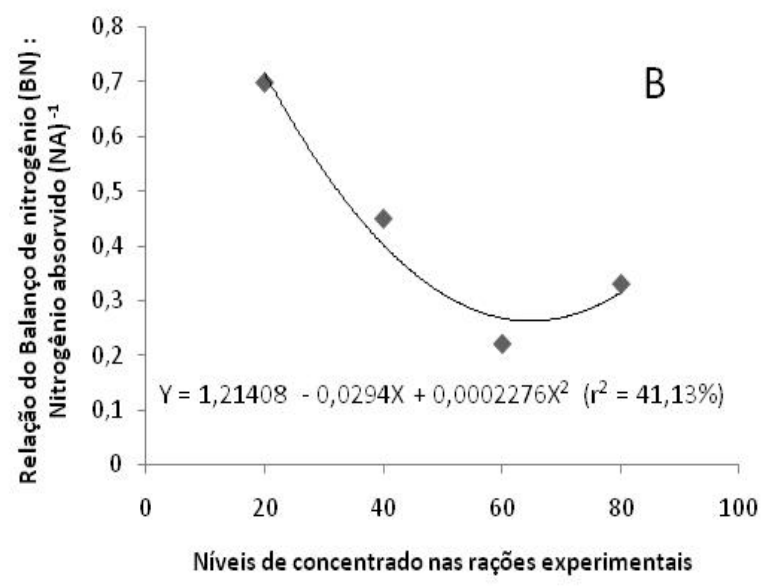

\section{Agradecimentos}

A Fundação de Amparo à Pesquisa do Estado de Mato Grosso (FAPEMAT) por ter concedido bolsas de iniciação cientifica aos acadêmicos do Curso de Zootecnia. A Universidade do Estado de Mato Grosso (UNEMAT) - Campus Universitário de Pontes e Lacerda, por ter apoiado e auxiliado no desenvolvimento desta pesquisa cedendo as instalações do Setor de Metabolismo Animal (SeMA) e equipamentos do Laboratório de Análise de Alimentos e Nutrição Animal (LAANA). Ao senhor e senhora Bragatto da fazenda Pau D'Alho por ter fornecido os animais (cordeiros) para execução do presente estudo. 


\section{Referências}

BERCHIELLI, T. T.; PIRES, A. V.; OLIVEIRA, S. G. Nutrição de ruminantes. Jaboticabal: Funep, 2011. 616 p.

BRINGEL, L. M. L.; NEIVA, J. N. M.; ARAÚJO, V. L.; BOMFIM, M. A. D.; RESTLE, J. FERREIRA, A. C. H.; LÔBO, R. N. B. Consumo, digestibilidade e balanço de nitrogênio em borregos alimentados com torta de dendê em substituição à silagem de capim-elefante. Revista Brasileira de Zootecnia, Viçosa, MG, v. 40, n. 9, p. 19751893, 2011.

DAMASCENO, J. C.; SANTOS, G. T.; CECATO, U.; SAKAGUTI, E. S.; ALCALDE, C. R.; BRANCO, A. F. Consumo voluntário, digetibilidade e balanço de nitrogênio em ovinos recebendo palha de arroz amonizada em diferentes níveis de oferta. Revista Brasileira de Zootecnia, Viçosa, MG, v. 29, n. 4, p. 1167-1173, 2000.

EZEQUIEL, J. M. B.; SAMPAIO, A. A. M.; SEIXAS, J. R. C.; OLIVEIRA, M. M. Balanço de nitrogênio e digestão total da proteína e da energia de rações contendo farelo de algodão, levedura de cana-de-açúcar ou uréia em ovinos. Revista Brasileira de Zootecnia, Viçosa, MG, v. 29, n. 6, p. 2332-2337, 2000. Suplemento 2.

FU, C. J.; FELTON, E. E. D.; LEHKUHLER, J. W.; KERLEY, M. S. Ruminal peptide concentration required to optimized microbial growth and efficiency. Journal of Dairy Science, Champaign, v. 79, n. 5 p. 1305- 1314, 2001.

GERON, L. J. V.; MEXIA, A. A.; CRISTO, R. L.; GARCIA, J.; CABRAL, L. S.; TRAUTAMANN, R. J.; MARTINS, O. S.; ZEOULA; L. M. Consumo, digestibilidade dos nutrientes e características ruminais de cordeiros alimentados com níveis crescentes de concentrado em ambiente tropical no Vale do Alto Guaporé - MT. Semina: Ciências Agrárias, Londrina, v. 34, n. 5 p. 2497-2510, 2013.

GERON, L. J. V.; MEXIA, A. A.; GARCIA, J.; SILVA, M. M.; ZEOULA, L. M. Suplementação concentrada para cordeiros terminados a pasto sobre custo de produção no período da seca. Semina: Ciências Agrárias, Londrina, v. 33, n. 2, p. 797-808, 2012a.

GERON, L. J. V.; MEXIA, A. A.; GARCIA, J.; ZEOULA, L. M.; GARCIA, R. R. F.; MOURA, D. C. Desempenho de cordeiros em terminação suplementados com caroço de algodão (Gossypium hirsutum L.) e grão de milho moído (Zea mays L.). Archives of Veterinary Science, Curitiba, v. 17, n. 4, p. 34-42, 2012 b.

KOZLOSKI, G. V. Bioquímica dos ruminantes. Santa Maria: UFSM, 2002. 140 p.
MENEZES, D. R. Utilização do farelo de mamona na alimentação de cordeiros em terminação. 2011. Tese (Doutorado em Zootecnia) - Centro de Ciências Agrárias, Universidade Federal da Paraíba, Areia.

MIZUBUTI, I. Y.; RIBEIRO, E. L. A.; ROCHA, M. A.; MOREIRA, F. B.; KHATOUNIAN, C. A.; PEREIRA, E. S.; FERNANDES, W. C.; SOUZA, L. W. O.; PINTO, A. P. Consumo médio e digestibilidade do feno de capim Coast Cross (Cynodon dactylon L. ) e feijão guandu (Cajanus cajan L. ) em carneiros submetidos a dosis regimes alimentares. Semina: Ciências Agrarias, Londrina, v. 28, n. 3, p. 513-520, 2007.

MORENO, G. M. B.; SILVA SOBRINHO, A. G.; LEÃO, A. G.; LOUREIRO, C. M. B.; PEREZ, H. L.; ROSSI, R. C. Desempenho, digestibilidade e balanço de nitrogênio em cordeiros alimentados com silagem de milho ou canade-açúcar e dois níveis de concentrado. Revista Brasileira de Zootecnia, Viçosa, MG, v. 39, n. 4, p. 853-860, 2010.

NUTRIENTS REQUIREMENTS OF SHEEP - NRC. Nutrients requirements of sheep. 5. ed. Washington, D. C.: National Academy Press, 1985. 99 p.

SANTOS, F. A. P.; PEDROSO, A. M. metabolismo de proteínas. In: BERCHIELLI, T. T.; PIRES, A. V.; OLIVEIRA, S. G. Nutrição de ruminantes. 2. ed. Jaboticabal: Funep, 2011. p. 265-292.

SILVA, D. J.; QUEIROZ. A. C. Análise de alimentos: métodos químicos e biológicos. 2. ed. Viçosa, MG: UFV, 2002. 178 p.

SILVA, J. F. C.; LEÃO, M. I. Fundamentos de nutrição de ruminantes. Piracicaba: Livroceres, 1979. 380 p.

SOUZA, E. Q. Panorama do mercado e os desafios de marketing na ovinocultura. Aguas de Lindóia: Associação Brasileira de Zootecnia. 2009. Disponível em: <http://www. abz.org.br/publicacoes-tecnicas/anaiszootec/palestras/22244-Panorama-mercado-desafiosmarketing-ovinocultura. html $>$. Acesso em: 18 maio 2013.

TIBO, G. C.; VALADARES FILHO, S. C.; COELHO DA SILVA, J. F.; VALADARES, R. F. D.; LEÃO, M. I.; CECON, P. R.; SAMPAIO, R. L. Níveis de concentrado em dietas de novilhos mestiços F1 Simental x Nelore. 2: Balanço nitrogenado, eficiência microbiana e parâmetros ruminais. Revista Brasileira de Zootecnia, Viçosa, MG, v. 29, n. 3, p. 921-929, 2000.

UNIVERSIDADE FEDERAL DE VIÇOSA - UFV. Sistema de análises estatísticas e genéticas - SAEG Viçosa: UFV, 1997. 150 p. (Manual do usuário). 
VALADARES FILHO, S. C.; MAGALHÃES, K. A.; ROCHA JÚNIOR, V. R.; CAPELLE, E. R. Tabelas brasileiras de composição de alimentos para bovinos. 2 . ed. Viçosa: UFV, 2006. 329 p.

VAN SOEST, P. J. Nutritional ecology of the ruminant. 2. ed. London: Constock Publishing Associates, 1994. 476 p.

VAN SOEST, P. J.; ROBERTSON, J. B.; LEWIS, B. A. Methods for dietary fiber neutral detergent fiber, and no starch polysaccharides in relation to animal nutrition. Journal of Dairy Science, Champaing, v. 74, n. 12, p. 3583-3597, 1991.
ZEOULA, L. M.; CALDAS NETO, S. F.; GERON, L. J. V.; MAEDA, E. M.; PRADO, I. N.; DIAN, P. H. M. Substituição do milho pela farinha de varredura de mandioca (Manihot esculenta, Crantz) em rações de ovinos: consumo, digestibilidade, balanços de nitrogênio e energia e parâmetros ruminais. Revista Brasileira de Zootecnia, Viçosa, MG, v. 32, n. 2, p. 491-502, 2003.

ZEOULA, L. M.; FERELI, F.; PRADO, I. N.; GERON, L. J. V.; CALDAS NETO, S. F.; PRADO, O. P. P.; MAEDA, E. M. Digestibilidade e balanço de nitrogênio com diferentes teores de proteína degradável no rúmen e milho como fonte de amido em ovinos. Revista Brasileira de Zootecnia, Viçosa, MG, v. 35, n. 5, p. 2179-2186, 2006. 